\title{
diffusion-Fundalarnentals.org
}

The Open-Access Journal for the Basic Principles of Diffusion Theory, Experiment and Application

\section{A Combined Atomistic Simulation and Quasielastic Neutron Scattering Study of the Low-Temperature Dynamics of Hydrogen and Deuterium Confined in NaX Zeolite}

\author{
Evangelia Pantatosaki, † George K. Papadopoulos, $\neq+$ Hervé Jobic, $†$ Doros $N$. \\ Theodorou, $f$
}

$\dagger$ Institut de Recherches sur la Catalyse et l' Environment, CNRS, 2 avenue Albert Einstein, 69626 Villeurbanne, France.

\$ School of Chemical Engineering, National Technical University of Athens, 9 Heroon Polytechniou Street, 15780 Athens, Greece.

\section{Introduction}

Atomistic MD simulation can address comparable time and length scales of molecular motion as the QENS technique [1,2]; they are, therefore, complementary and can form a basis for illuminating comparisons. In modeling hydrogen and deuterium confined within zeolite pores, a classical treatment of the energetics is not entirely satisfactory. In this work we carry out a simultaneous study of the sorption thermodynamics and kinetics of hydrogen and deuterium in the zeolite $\mathrm{NaX}$ by means of QENS experiments and equilibrium MD simulations, taking into account the quantum mechanical effects.

\section{Experimental Method}

The adsorption isotherm of $\mathrm{H}_{2}$ in $\mathrm{NaX}$ at $77 \mathrm{~K}$ was measured volumetrically by means of an in-house built apparatus. The quasielastic neutron scattering experiments were carried out at the ILL (Institut Laue-Langevin, Grenoble, France) to determine the self $\left(D_{s}\right)$ and transport $\left(D_{t},\right)$ diffusion coefficients for $D_{2}$ in NaX. Taking into account the data treatment and the values from refinements using different jump diffusion models [3], typical mean errors of $20 \%$ and $50 \%$ were estimated for $D_{s}$ and $D_{t}$, respectively.

\section{Simulation Method}

For all sorbate-sorbate and sorbate-sorbent atom interactions Feynman's effective quadratic potential, $U_{\mathrm{q}},[4,5]$ was used

$$
U_{q}\left(r_{i j}\right)=U_{\mathrm{LJ}}\left(r_{i j}\right)+\frac{\beta \hbar^{2}}{24 m_{\mathrm{r}}}\left[\frac{\partial^{2} U_{\mathrm{LJ}}\left(r_{i j}\right)}{\partial r_{i j}{ }^{2}}+\frac{2}{r_{i j}} \frac{\partial U_{\mathrm{LJ}}\left(r_{i j}\right)}{\partial r_{i j}}\right]
$$

where $r_{i j}$ is the position vector distance between the centers of mass of molecules $i$ and $j$. In place of the atomic mass, the reduced mass, $m_{\mathrm{r}}$, must be used.

To reconstruct a digital $\mathrm{NaX}$ crystal, the unit cell geometric characteristics, X-Ray diffraction data and the symmetry operations of the relevant space group were used to obtain the position vectors of all framework atoms [6]. The simulated equilibrium sorption experiments of $\mathrm{H}_{2}$ and $\mathrm{D}_{2}$ in $\mathrm{NaX}$ were performed by employing a Grand Canonical Monte Carlo (GCMC) algorithm. Equilibrium molecular dynamics (MD) 
simulation was carried out in the microcanonical (NVE) ensemble; the self, transport and collective diffusion coefficients were calculated [5].

\section{Results and Discussion}

The experimental and simulated self diffusion coefficients for $\mathrm{H}_{2}$ and $\mathrm{D}_{2}$ are compared (Figure 1a), showing an increase of the self diffusivity as the loading increases; this can be explained on the basis of calculating, via GCMC, the partial molar configurational internal energy of the sorbed phase [5]. In Figure 1b, it is shown that the strongest sorption sites with lower energy inside the zeolite pores are firstly populated by the sorbate molecules, while as the loading increases the new sorbed molecules are free to move inside pores.

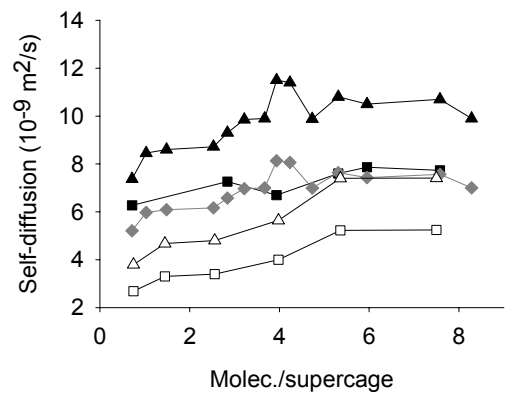

(a)

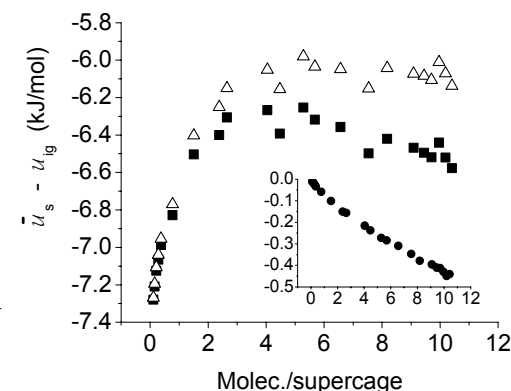

(b)

Figure 1. (a) Self diffusivities measured by QENS for $\mathrm{H}_{2}$ (open triangles) and $\mathrm{D}_{2}$ (open squares) and computed by MD (filled symbols) as a function of their loading in NaX; (b) Partial molar configurational internal energy of sorbed phase calculated for $\mathrm{H}_{2}$ via GCMC simulation vs. loading, shown as total (filled squares), and separated into sorbate-sorbent (open triangles) and sorbatesorbate interactions (inset).

Computed and experimental transport diffusivities exhibit a very large increase with the loading and are in very good agreement, except for higher loadings [5]. This discrepancy is expected, since in our calculations the experimental and simulated isotherms exhibit different saturation values. It also appears that the corrected diffusivity is not constant, contrary to the assumption often made in the interpretation of macroscopic data.

\section{References}

[1] Jobic, H.; Theodorou, D. N. Micropor. Mesopor. Mater. 102 (2007) 21.

[2] Papadopoulos, G. K.; Jobic, H.; Theodorou, D. N. J. Phys. Chem. B 108 (2004) 12748.

[3] Jobic, H.; Kärger, J.; Bée, M.; Phys. Rev. Lett. 82 (1999) 4260.

[4] Feynman, R.P.; Hibbs, A.R. Quantum Mechanics and Path Integrals, McGraw-Hill, New York, 1965.

[5] Pantatosaki, E.; Papadopoulos, G.K., Jobic H., Theodorou D. N. J. Phys. Chem. B 112 (2008) 11708.

[6] Pantatosaki, E.; Papadopoulos, G.K., J. Chem. Phys. 127 (2007) 164723. 